\title{
Five Y Cable and a Single GPRS Device Transmitted the Real Time Environmental Data from AAQMS
}

\author{
Siba P. Panda, Jay Rath, A. K. Swar, Sidhanta Das, and Ranjita Panda
}

\begin{abstract}
Usually AAQ (Ambient Air Quality) station consists of more than one analyzer. Connecting Y Cable and GPRS device in each analyzer for transmitting data to regulatory authority is highly expensive. This led to a bottleneck as each Y Cable needs a separate GPRS (General Packet Radio Service) device to transmit the data from each analyzer to the regulatory authority server over GPRS link. This paper highlights capturing data from five analyzers using five $Y$ cables and a single GPRS device to transmit all five analyzer's data to the destination server.
\end{abstract}

Index Terms-ASCII (American Standard Code for Information Interchange), DSR (Data Set Ready), GPRS (General Packet Radio Service), PCI (Peripheral Component Interconnect).

\section{INTRODUCTION}

Online monitoring system always connects with a local PC with customized software to monitor the environmental data. The connection between the local PC and the analyzer can be done using a RS232 cable. The customized software installed in the local PC initiates a request to the analyzer to send the environmental data periodically. The environmental data after arrival can be saved in a database or any file in the local PC for monitoring purpose. Periodic report can be generated to submit to the regulatory authority for obtaining the consent to operate certificate from the saved data.

This report usually dispatched to regulatory authority by post. Hence the regulatory authority always receives the data after two or three days from the date of report generation. Report form was not a real time data and the genuineness of data was questionable.

Since the data was available easily in local PC, the industry has the option to decrease the value of each parameter to satisfy the regulatory terms prescribed by the regulatory authority. Hence in summary, the data arrived at regulatory authority in for clarity sake, if the threshold value for PM10 (Particulate Matter of 10 microns diameter size) is prescribed as $100 \mu \mathrm{g} / \mathrm{m}^{3}$ and if the data arrived from analyzer exceeds the threshold value, to avoid all the hassle the industry can manipulate the data to match the threshold value.

Manuscript received October 10, 2013; revised December 1, 2013.

Siba P. Panda is with Env. Head, Hindalco Industries Limited; Hirakud, Sambalpur, India (e-mail:siba_panda@yahoo.com).

Jay Rath is with Sunjray Pty. Ltd, Sydney, Australia (email: jay.rath@sunjray.com).

A. K. Swar and Sidhanta Das are with State Pollution Control Board; Bhubaneswar, Odisha, India (e-mail: swarakhila@gmail.com).

Ranjita Panda is with P.G. Department of Environment Science; Sambalpur University, India (e-mail: ranjita_panda@yahoo.co.in).

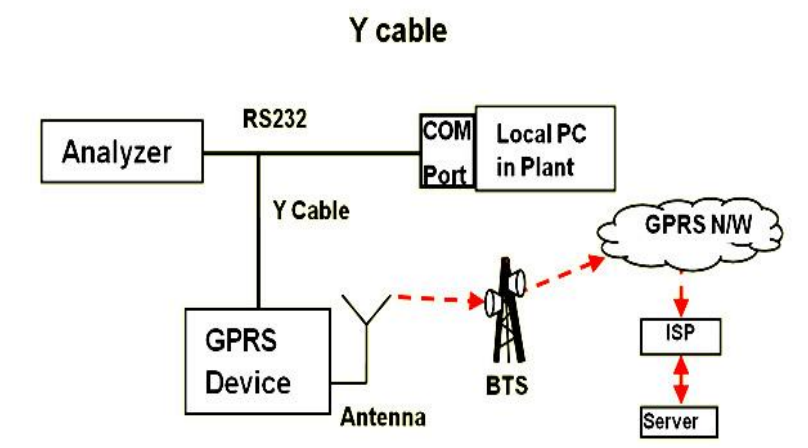

Fig. 1. Y Cable connection with PM10 analyzer and a single GPRS device.

This was a big challenge for regulatory authority to get the right data in right time. Hence the $\mathrm{Y}$ cable was introduced to capture the analyzer output that goes to the local PC. It was a major breakthrough for State Pollution Control Board, Odisha back in September 2012. Using Y cable the analyzer data was captured and transmitted to the server over GPRS link. This satisfies the arrival of data on real time and the genuine data from the analyzer. Fig. 1 shows the capture of data using Y cable and transmission of the same data to the server [1].

Y cable was a great success while capturing data from a single analyzer and transmitting the data to the destination server using a single GPRS device over GPRS link. However, the AAQ station usually installed with five different analyzers to measure the parameter such as PM2.5, $\mathrm{PM} 10, \mathrm{CO}, \mathrm{SO}_{2}$ and $\mathrm{NO} 2$. In case of capturing data from more than one analyzer, a single $\mathrm{Y}$ Cable is not the right answer. Hence multiple Y Cable can be extended from each analyzer's output (RS232 connection) and combining all the output of Y Cables to a single GPRS device for transmission of all five analyzer's data to the destination server over GPRS network [2].

\section{DifFERENT Methods OF CAPTURING DATA FROM ANALYZER}

\section{A. Capturing Analog Output from Each Analyzer}

Basically the analyzer has different types of output option such as output in RS232 format, 4-20ma format, 0-1 Volt format, 0-10Volt format and RJ45 format. Considering the cost, most of the industry opts for a single type adaptor. It might be RS232 or 4-20ma etc that depends on their budget. Some industries do not choose for RS232 or RS485 output as well. Rather the output is provided in form of analog signal such as 4-20ma or 0-1 Volt or 0-10 Volt.

In case of analog output, a converter should be connected to convert the analog signal to interpret the analog value in 
digital format. Converting the analog signal to interpret in digital format needs extra devices which enhances cost compared to the direct RS232 output from analyzer. However, the analog output from all five analyzers can be connected to a converter to interpret the analog value in digital format for connecting to a single GPRS device for transmission to the destination server. This type of capturing data from analyzer is quite possible. But it is difficult to convince industries to install extra adaptor for RS232 output if it is not present in each analyzer. Rather the industry insists on capturing output from analog output for onward transmission to the destination server over GPRS link. The figure (Fig. 2) shows the capture of analog output from all five analyzers and using two different converters the final output is connected to a single GPRS device for transmission of all five analyzer's data to the destination server.

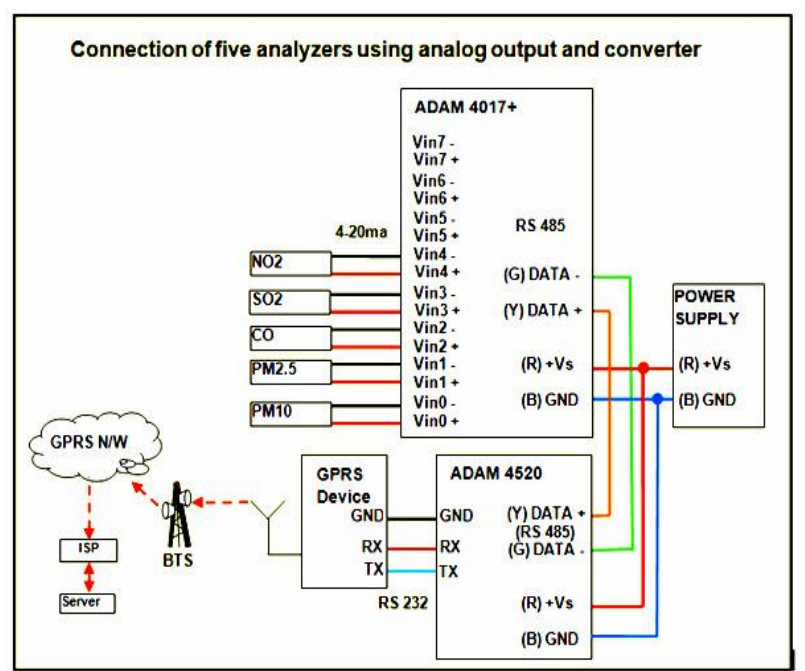

Fig. 2. Capturing five analyzer's analog output and transmitting the data to server after conversion.

\section{B. Capturing Output from RS232 Port of Each Analyzers}

The output from each analyzer is connected with an Octopus cable with DB62 connector at the PC end for communication. The DB62 connector is attached with a PCI controller card which is inserted in PC internally. Hence, it is not easy to get a single COM port output (COM Port with one RXD one TXD and one GND signal) at the PC end with all the five analyzer's data. Hence separate Y Cables from each RS232 cable that connects with the output of individual analyzer and the local PC have to be extended. The other end of five $\mathrm{Y}$ cables should be connected with five RS232 port of a MOXA converter. The MOXA converter can have multiple RS232 ports for data input and a single RS232 port for output.

The output from MOXA can be connected to a COM Port of a PC to receive the data from all five analyzers. A customized program runs in the PC with two threads. The first thread is to receive the data from all five analyzers through the MOXA converter and the second thread is used to write the data to a second COM port in the PC for communicating with the GPRS device.

The Fig. 3 shows the capture of data from all five analyzers using five separate Y Cables and tunneling all five output data to a single output in RS232 port of MOXA for communicating with the PC. Finally the data can be transmitted to the destination server using a GPRS device connected in the COM port of the PC. This method is not appropriate as it's expensive and dependant on the connected PC.

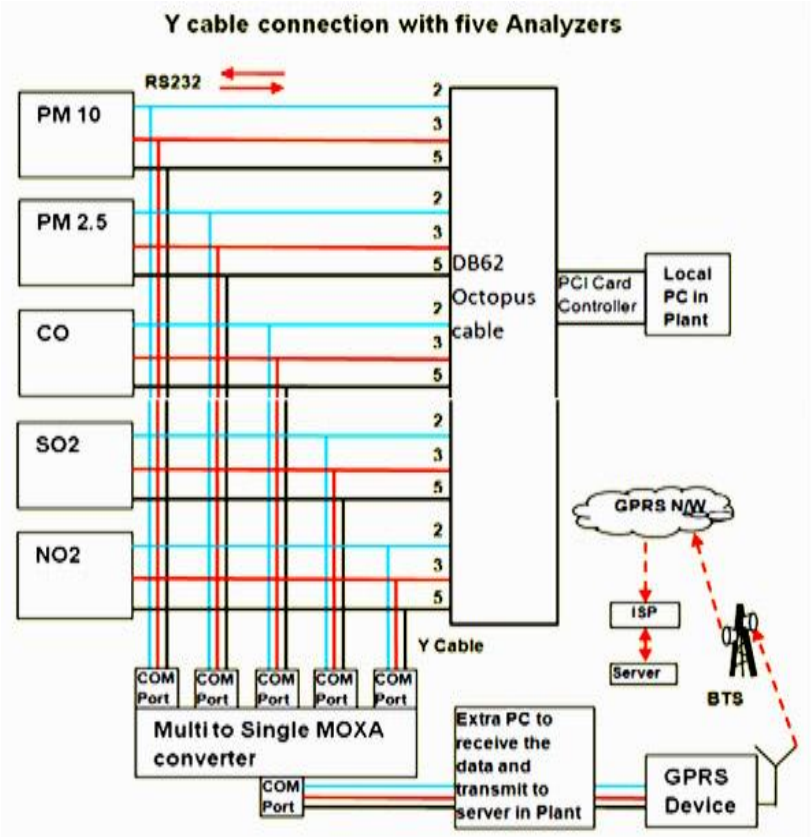

Fig. 3. Y cable connection with five analyzers.

\section{Capturing Output from RS232 Port of Each Analyzers Using Daisy Chain}

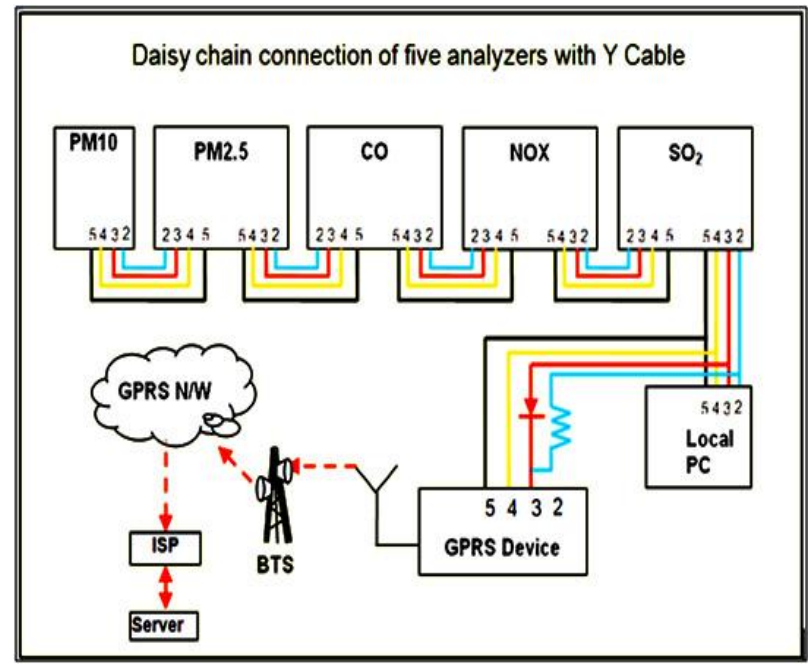

Fig. 4. Daisy chain connection of five analyzers with a Y Cable.

Certain industries connect their analyzer in a series format. In this method the output comes in a cyclic manner. The output from PM10 analyzer is connected to the PM2.5 analyzer. Subsequently the output from PM2.5 analyzer is connected with the $\mathrm{CO}$ analyzer in a daisy chain manner. RX, TX, GND and CTS pins are connected through out. The $\mathrm{SO} 2$ analyzer output is connected to the COM port of the local PC with RX, TX, GND and CTS wiring. The local PC has customized software running to accept the parameter value periodically from each analyzer. After receiving the parameter value from the analyzers the data has been saved in the local PC for future reference. However, the data does 
not arrive from all five analyzers in every time slot. Rather it arrives in a cyclic manner. For clarity sake, in T1 time period PM10 can send data and the rest four analyzers do not send data. Similarly in T2 time slot, the PM2.5 analyzer sends data and the rest analyzers do not send data. Using a single Y cable, all the five analyzers output can be captured. It takes five time slots to get the output from each analyzer. This is one of the convenient ways of capturing analyzer output data that goes to local PC. However, most of the industries do not use this type of connection. Hence changing the existing set up from daisy chain to parallel connection is beyond question.

The above mentioned three types of connection to capture the data from all five analyzers are not suitable as it involves more cost and lot of change is required to change the existing set up. This leads to a bottleneck situation to capture and transmit data from all five analyzer's RS232 Port using five Y Cables and a single GPRS device. Hence this paper highlights the test that has been carried out in different industries for capturing data using five $\mathrm{Y}$ cables with a single GPRS device [3]-[7].

\section{Y CABLE DESIGN}

RS232 (Recommended Standard 232) is the standard for serial data communication between DTE (Data Terminal Equipment) and DCE (Data Circuit-terminating Equipment). A typical Data Terminal Equipment is a computer and a typical Data Communications Equipment is a Modem. The length of the cable between DTE and DCE should not be more than 50 feet unless a signal booster is used. Most commonly used types of serial cable connectors are 9-pin connector which is called as DB9. Similarly the 25-pin connector is called as DB25. It can be female or male type connector. The Table below describes the detailed PIN present in RS232 cable.

TABLE I: PIN NUMBER, NAME AND DESCRIPTION OF DB9 RS232 CABLE

\begin{tabular}{|c|c|c|}
\hline PIN\# & Acronym & Name \\
\hline 3 & TXD & Transmit Data(Serial data Output) \\
\hline 2 & RXD & Receive data(Serial data input) \\
\hline 7 & RTS & $\begin{array}{c}\text { Request to Send(acknowledge to modem } \\
\text { that UART is ready to exchange data) }\end{array}$ \\
\hline 8 & CTS & $\begin{array}{c}\text { Clear to Send(MODEM is ready to } \\
\text { exchange data) }\end{array}$ \\
\hline 6 & DSR & Data Set Ready(UART establish a link) \\
\hline 4 & DTR & Data Terminal Ready \\
\hline 1 & DCD/CD & $\begin{array}{c}\text { Data Camera Detect(This Line is active } \\
\text { when MODEM detects a carrier) }\end{array}$ \\
\hline 9 & RI & $\begin{array}{r}\text { Ring Indicator(Becomes active when } \\
\text { MODEM detects ringing signal from PSTN) }\end{array}$ \\
\hline 5 & SG & Signal Ground \\
\hline
\end{tabular}

Only three pins such as TXD, RXD and GND Pins are used for transmit and receive data between the DTE and DCE. All other pins can be controlled by software and can be used for other purposes. In case of LOW signal the voltage value in the pin should be between +3 Volts to +12 Volts. This is represented as 0 or LOW signal. Similarly, for high signals the voltage value should be between -12 Volts to -3 Volts. This is represented as 1 or HIGH signal (see Table II).
TABLE II: DIRECTION OF SIGNALS FROM EACH PIN IN A DB9 RS232

\begin{tabular}{|l|l|l|l|l|}
\hline PIN\# & Acronym & Name & Path & Description \\
\hline 3 & TXD & $\begin{array}{l}\text { Transmit } \\
\text { Data }\end{array}$ & $\longrightarrow$ & $\begin{array}{l}\text { Transmit bytes out } \\
\text { of computer or } \\
\text { device }\end{array}$ \\
\hline 2 & RXD & $\begin{array}{l}\text { Receive } \\
\text { Data }\end{array}$ & $\longleftarrow$ & $\begin{array}{l}\text { Receives bytes into } \\
\text { computer or device }\end{array}$ \\
\hline 7 & RTS & $\begin{array}{l}\text { Request } \\
\text { To Send }\end{array}$ & $\longrightarrow$ & $\begin{array}{l}\text { RTS/CTS Flow } \\
\text { Control }\end{array}$ \\
\hline 8 & CTS & $\begin{array}{l}\text { Clear to } \\
\text { Send }\end{array}$ & $\longleftarrow$ & $\begin{array}{l}\text { RTS/CTS Flow } \\
\text { Control }\end{array}$ \\
\hline 6 & DSR & $\begin{array}{l}\text { Data Set } \\
\text { Ready }\end{array}$ & $\begin{array}{l}\text { Data } \\
\text { Terminal } \\
\text { Ready } \\
\text { to communicate }\end{array}$ \\
\hline 1 & DTR & $\begin{array}{l}\text { Data } \\
\text { Carrier } \\
\text { Detect }\end{array}$ & $\longleftarrow$ & $\begin{array}{l}\text { The device is ready } \\
\text { to communicate }\end{array}$ \\
\hline 9 & RI & $\begin{array}{l}\text { Ring } \\
\text { Indicator } \\
\text { Signal } \\
\text { Ground }\end{array}$ & $\longleftarrow$ & \begin{tabular}{l} 
Modem connected \\
to another \\
\hline 5
\end{tabular} \\
\hline
\end{tabular}

Hardware flow control can be used if the RTS and CTS and DTR and DSR pins are connected.

When RTS set to LOW, the DTE stop sending data. The LOW state represents -12 Volts and it is an indication to stop sending data. Similarly in case of RTS the state changes to HIGH and the output voltage become +12 Volts. In this case the data start flowing to computer.

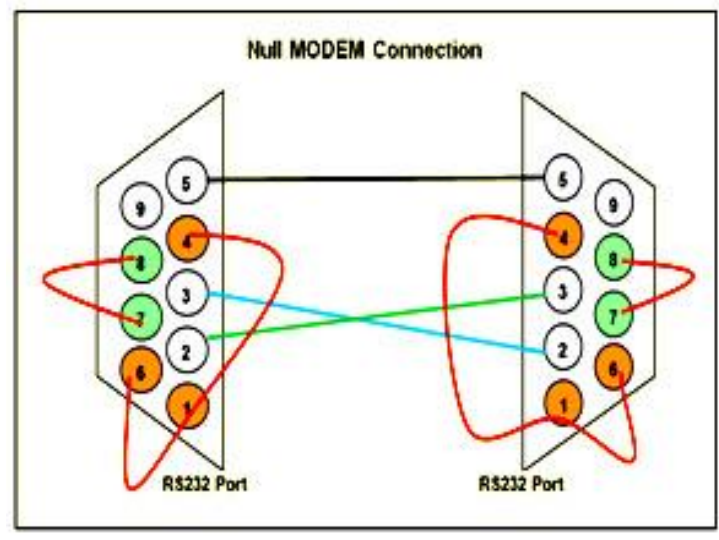

Fig. 5. Null modem connection.

Null modem is used to transfer files between the computers using protocols such as Zmodem protocol, xmodem protocol, etc.

Fig. 5 shows the wiring of the null modem. The guest \& host computer can be connected through the TXD, RXD, and GND pins. The data can be transmitted through TXD line from the Host to Guest and is received on RXD line of guest. Both signal ground (GND) pin must be connected to each other to have a common ground. The Data Terminal Ready (DTR) pin (PIN 4) can be looped back with Data Set Ready (DSR) pin (PIN 6) and Data Carrier Detect (DCD) or Carrier Detect (CD) pin (PIN 1) on both computers. When the Data Terminal Ready is active, the Data Set Ready and Carrier Detect also change to active state. At this point, the computer assumes that the Modem is ready. Hence the computer starts sending data as long as the DTR is active. Request to Send (RTS) pin (PIN 7) and Clear To Send (CTS) pin (PIN 8) can be connected together to make the computer assume that it is OK to send the data when RTS 
turns to HIGH. The table shown below describes the pin connection for loopback purpose. A loopback test can be used to troubleshoot serial communications. It can show errors in the serial port or errors in the serial cable without connecting any analyzer or hardware devices.

TABLE III: THE LOOPBACK CONNECTION OF RS232 PINS

\begin{tabular}{|c|c|c|}
\hline \multicolumn{3}{|c|}{ NULL MODEM CABLE } \\
\hline DB9 & & DB9 \\
\hline Pin\# & Connects to & Pin\# \\
\hline 1 & $4 \rightarrow \rightarrow$ & 4 \\
\hline 2 & $4 \rightarrow$ & 3 \\
\hline 3 & $4 \rightarrow$ & 2 \\
\hline 4 & $4 \rightarrow$ & 1,6 \\
\hline 5 & $4 \rightarrow$ & 5 \\
\hline 6 & $4 \rightarrow$ & 4 \\
\hline 7 & $4 \rightarrow$ & 8 \\
\hline 8 & & 9 \\
\hline
\end{tabular}

Basing on RS232 pin connection and signal flow principle, the Y Cable concept has been developed (see Fig. $6)$.

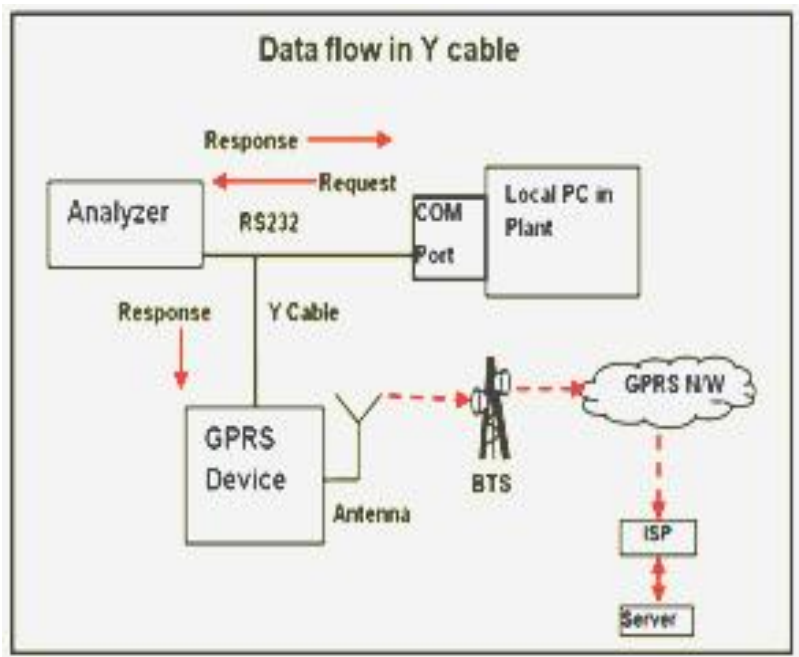

Fig. 6. Basic Y cable concept.

However, it is clearly instructed by the regulatory authority not to disturb the existing set up between analyzer and the local PC [1].

The local PC sends the initial request signal to the analyzer using PIN 3. After receiving the request signal, the analyzer responds to the local PC using PIN 2. This goes on continuously. Regarding capturing the data that goes to local PC, we need to extend another connection from PIN 2, PIN 3 and PIN 5 which is termed as Y cable. Cross cable is used to connect a GPRS device. Hence PIN 3 of GPRS device receives data like PIN 2 of local PC. PIN 2 of GPRS device should send the request to analyzer like PIN 3 of local PC. But since local PC is sending the request and getting the response, the request from GPRS device to analyzer is blocked.

However, during the respond cycle, the data goes from analyzer to local PC and the same data is diverted to the GPRS device. To initiate the flow control, the pin 4 should be connected with pin 6 and pin 1 of the GPRS device. Similarly pin 7 and pin 8 should be connected. Hence the GPRS device presumes that the request is being sent and the respond is coming to the GPRS device (see Fig. 7).

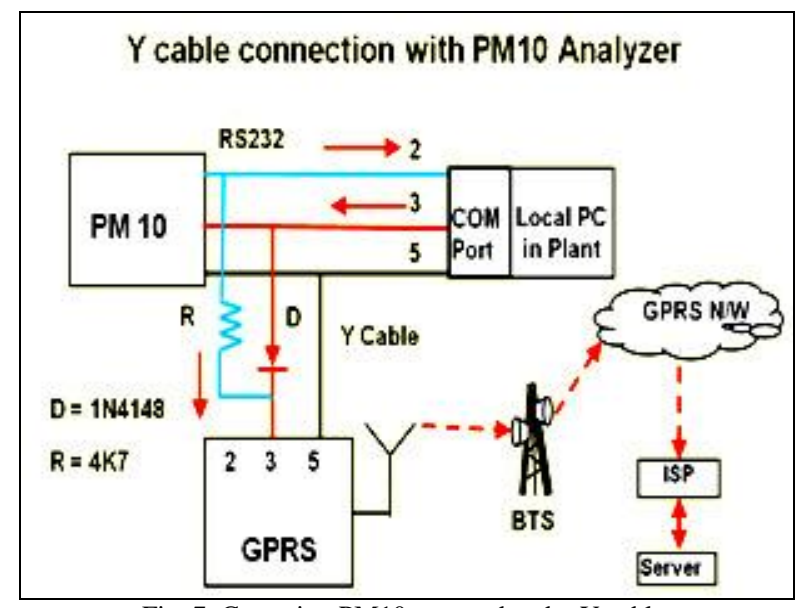

Fig. 7. Capturing PM10 output data by Y cable.

\section{Multiple Y CABle With a Single GPRS DEVICE}

Using five $\mathrm{Y}$ cables and a single GPRS device, the test was carried out in a plant in Orissa. The test was successful by capturing the data from all five analyzers and transmitting all five analyzers data to destination server using a single GPRS device.

The Fig. 8 shown below shows the detailed diagram of connection. The AAQ station was installed with five different analyzers to measure PM10, PM2.5, $\mathrm{SO}_{2}, \mathrm{NOX}$ and $\mathrm{CO}$ parameters value in a regular basis. All those five analyzers were connected with RS232 interface with the local PC separately using transparent serial communication protocol.

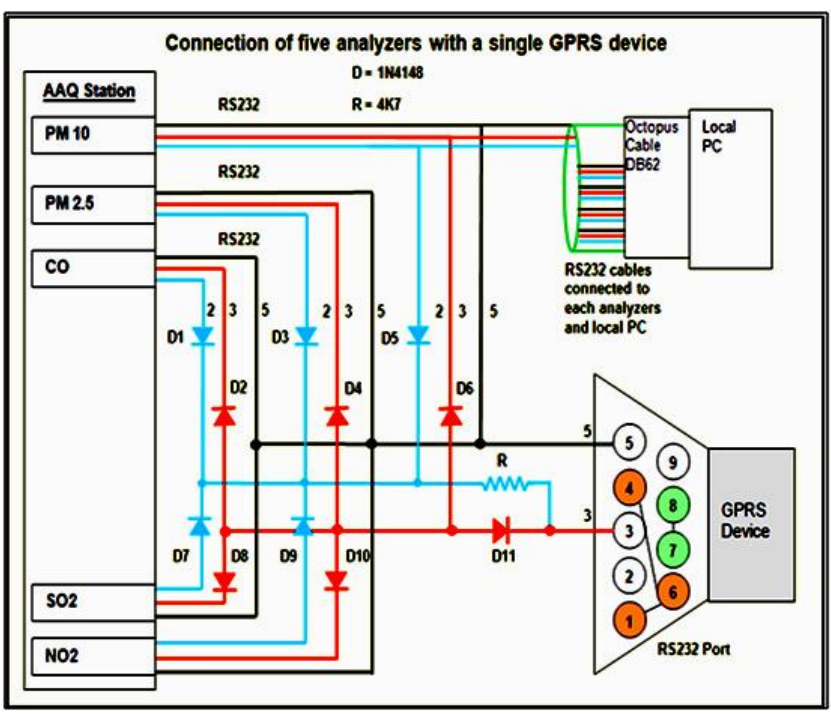

Fig. 8. Five Y cables from five analyzers are connected to a single GPRS device for onward transmission.

The output from each analyzer is connected with an Octopus cable with DB62 connector at the PC end. However, the cable was attached with a PCI controller card which was inserted in PC internally. Hence, it is not easy to get a single COM port output (COM Port with one RXD one TXD and one GND signal) at the PC end. Hence, it was decided to extend $\mathrm{Y}$ cable from each RS232 cable connected between analyzer and the local PC. Later all the PIN2 and PIN3 of Y cable were connected to a single GPRS Device.

RS232 cables with RX, TX and GND signals are connected between the DB62 end (Local PC) and the 
analyzer end. Y Cable was connected to carry the same RX, TX and GND signals from each analyzer to the other end of Y cable. PIN 2, PIN 3 and PIN 5 of $Y$ cables were connected separately. The PIN2 of each Y Cable is connected to a single point with a resistor at the end. The resistor value was $4 \mathrm{~K} 7$. Similarly PIN 3 of each $\mathrm{Y}$ cable is connected to a single point with a diode $(1 \mathrm{~N} 4148)$ at the end to block the reverse current. The negative end (Cathode) of diode and the resistor were connected to PIN 3 of GPRS device. PIN 2 of GPRS device was not connected. With this basic circuit, the output from each analyzer was passed to PIN 3 of GPRS device.

PIN7 and PIN 8 of GPRS device are also loopback for allowing the output from all five analyzers to arrive at the GPRS Device. PIN 4, PIN 6 and PIN 1 are also connected together for flow control. The picture shown above (Fig. 7) is the detailed wiring diagram. At the destination server end we could see the data in a serial manner continuously [3]-[8].

\section{ANALYSIS OF OUTPUT}

The data arrived at the destination server from the AAQ station is displayed below. This data is displayed before any filtration.

Normal power on

GPRS DTU SUNJRAY_3.0.0.1

Address of server1 is 113.19.81.6:8313

Address of server2 is 0.0.0.0:0

Address of server 3 is $0.0 .0 .0: 0$

Address of server4 is 0.0.0.0:0

APN :(aircelgprs.com,,)

+WIND: 13

+CREG: 0

+CGREG: 0

Imei: 358948011083217

+WIND: 1

+CREG: 2

\section{9:45:11:}

$>+00.872+01.029+02.589+02.816+04.234+00.869+03.167+00.014$

19:45:12: M10416130913M00 51.7885 28.0026 16.6658 17

19:45:18: AF2216130913M00 3.93193600 5E

19:45:38: AC3216130913M00 2.7777 22.00151 20.01381 5E

19:45:59: CO1216130913M00 0.593768 2.717836e-20 3.491663e+14 57

19:46:00:

$>+00.872+04.321+02.593+02.816+04.222+00.869+03.167+00.014$

19:46:08: AF2216130913M00 3.953346 0057

19:46:09: M10416130913M00 51.7885 28.0026 16.6658 17

19:46:28: AC3216130913M00 2.661289 22.37223 19.71095 5B

19:46:29: CO1216130913M00 0.587495 2.717836e-20 3.491663e+14 53

19:47:00:

$>+00.872+01.830+02.593+02.814+04.206+00.869+03.167+00.014$

19:47:07: AF2216130913M00 3.915877 $005 \mathrm{C}$

19:47:07: AC3216130913M00 2.648447 22.13144 19.4829954

19:47:08: M10416130913M00 51.7885 28.0026 16.6658 17

19:47:19: CO1216130913M00 0.583035 2.717836e-20 3.491663e+14 59

19:48:08: AF2216130913M00 $3.856548005 \mathrm{~B}$

19:48:09: CO1216130913M00 0.58094 2.717836e-20 3.491663e+14 61

19:48:09:

$>+00.881+00.984+02.593+02.819+04.199+00.869+03.167+00.014$

19:48:20: M10416130913M00 51.7885 28.0026 16.6658 17

19:48:20: AC3216130913M00 2.666306 21.30145 18.63515 52

19:49:00:

$>+00.872+04.215+02.593+02.819+04.192+00.869+03.167+00.014$

19:49:08: AF2216130913M00 3.804401 0050

19:49:08: M10416130913M00 51.7885 28.0026 16.665817

19:49:28: AC3216130913M00 2.662381 20.54278 17.8804 68

19:49:29: CO1216130913M00 $0.5749312 .717836 \mathrm{e}-203.491663 \mathrm{e}+145 \mathrm{D}$
19:50:08: AF2216130913M00 3.80332300 00

19:50:08:

$>+00.872+04.107+02.593+02.820+04.206+00.869+03.167+00.014$ 19:50:08: M10416130913M00 51.7885 28.0026 16.6658 17

19:50:08: CO1216130913M00 0.577836 2.717836e-20 3.491663e+14 59

19:50:18: AC3216130913M00 2.634706 20.45556 17.82085 58

19:51:08: AF2216130913M00 3.81017300 05

19:51:08: AC3216130913M00 2.609138 20.12249 17.51335 50

19:51:09: M10416130913M00 51.788528.002616.6658 17

19:51:09:

$>+00.872+00.875+02.593+02.817+04.183+00.869+03.167+00.014$

19:52:00:

$>+00.872+04.110+02.593+02.819+04.171+00.869+03.168+00.014$

19:52:00: M10416130913M00 51.7885 28.002616.6658 17

19:52:01: CO1216130913M00 0.561725 2.717836e-20 3.491663e+14 53

19:52:08: AF2216130913M00 3.795706 0053

19:52:08: AC3216130913M00 2.590039 19.78641 17.19637 52

19:53:08: AF2216130913M00 3.7277150058

19:53:08: AC3216130913M00 2.506551 19.26851 16.76195 55

19:53:08: M10416130913M00 51.7885 28.0026 16.5267 1C

19:53:09:

$>+00.872+00.977+02.593+02.819+04.167+00.869+03.168+00.014$

19:53:29: CO1216130913M00 0.567755 2.717836e-20 3.491663e+14 52

Each analyzer has a unique ID such as M104 stands for PM10 analyzer. AF22 has been assigned as the $\mathrm{SO}_{2}$ analyzer ID. Similarly AC32 has been assigned for $\mathrm{NO}_{\mathrm{X}}\left(\mathrm{NO}, \mathrm{NO}_{\mathrm{X}}\right.$ $\mathrm{No}_{2}$ ) analyzer's ID. For $\mathrm{CO}$ analyzer, the ID has been assigned as CO12. The output from four analyzers is shown in red color except PM2.5 analyzer. PM2.5 analyzer's output is in form of 4-20ma format.

\section{CONCLUSION}

Basically, capturing output from five analyzers and transmitting through a single GPRS device is the best solution as it reduces the cost and efforts of writing extra program. The data can be captured using Y Cable from all the analyzers installed in AAQ stations and transmitted to the destination server without any delay and manipulation. In future the circuit should be developed for capturing output from eight different analyzers. Enhancing the capability for capturing more number of analyzer's output and transmitting the same with a single GPRS device can reduce the cost and efforts of writing extra program at front end.

\section{REFERENCES}

[1] S. P. Panda, J. Rath, A. K. Swar, and R. Panda, "Real time Ambient Air Quality (AAQ) and stack emission data transmission through Y cable mode over GPRS link," in Proc. International Conference on Chemical, Bio-Chemical and Environmental Sciences (ICBEE'2012), Singapore, December 14-15, 2012, pp. 27-30.

[2] Agilent Technologies, Inc. (June 28, 2001). Understanding general packet radio service (gprs), application note 1377. [Online]. Available: http://cp.literature.agilent.com/litweb/pdf/59882598EN.pdf

[3] RS232. [Online]. Available: http://en.wikipedia.org/wiki/RS-232

[4] UART. [Online]. Available: http://en.wikipedia.org/wiki/Universal_asynchronous_receiver/transm itter

[5] Sensor Network based on IEEE 1451.0 and IEEE p1451.2-RS232. [Online]. http://www.nist.gov/customcf/get_pdf.cfm?pub_id=824642

[6] RS-232 Utility, White Paper, WP-RS232UTIL, ver. 1.1, Altera Corporation, April 2003,

[7] Serial Programming/RS-232 Connections. [Online]. Available: http://en.wikibooks.org/wiki/Serial_Programming/RS232_Connections

[8] T. Halonen, J. Romero, J. Melero, GSM, GPRS and EDGE Performance: Evolution Towards 3G/UMTS, 2nd ed. John Wiley \& Sons Ltd., 2003. 


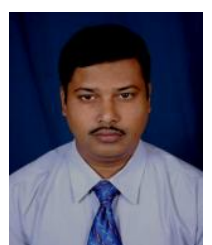

Siba Prasada Panda was born at cuttack, Odisha India on July 1, 1975. He did his M.Sc. in botany from Utkal University, Bhubaneswar Odisha, India in 1997 and ph.D. in environment science from Utkal University, Odisha, India in 2004. He has more than 16 years of work Experience in Industry, Research and Teaching in the field of Environmental Science. He has published more than 35 research articles in reputed national and International journals and presented more than 35 research papers in different national and international conferences. He has significantly contributed in the field of Environmental Sciences. He has research expertise in the field of Solid waste Management, Aerosol, EIA, Environment Biology, Bioinformatics, Environment Management, Environmental Biotechnology, Pollution Monitoring ,Natural Resource Conservation and Management, Waste Management etc. He has published a book "Natural Resource Conservation and Environment Management" APH, N.Delhi Publication. He has guided many M.Tech., M.Sc., B.Tech and Ph.D students. He has visited Thailand, Japan, Singapore et and chaired so many national and International Conferences.

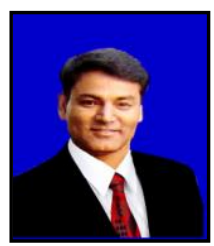

Jay Rath is an Indian and he was born in Australia with more than 20 years of experience in IT and Embedded Technology focusing mainly on project management, research and development of niche concepts. He has completed his bachelor in computer system engineering from Shivaji University in India in 1991 and did his Post Graduate diploma in Manufacturing Technology from University of South Australia, Australia in 1994. He has also completed his post graduation research in Telecommunication specializing in ATM (Asynchronous Transfer Mode) Switching from University of Adelaide, Australia in 1995. He is a certified SUN Microsystems, SAP and VIGNETT professional. He is a reputable $\mathrm{BI}$ advisor in Asia Pacific (APAC) region, experienced in Cognos, Business Object, Hyperion, Microstrategy, Elixir and PlumTree products. His vast experience on BI helped few companies to develop their customized BI products as well.

Currently he is holding the chief technical officer post of Sunjray Pty Ltd, Australia and also appointed as the chief solution architect for Sunjray Infosystems Pvt Ltd. India to deal with RT-DAS projects.

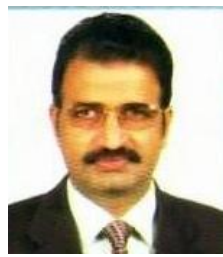

Akhila Kumar Swar is a senior environmental engineer of the State Pollution Control Board,Odisha,India, with a Ph.D. degree in Engineering from Sambalpur University (R.E.C. Rourkela as centre of Research),odisha,India,2003 and Masters in Environmental Engineering from the Asian Institute of Technology, Bangkok, Thiland, 1990. He has been working in the State Pollution Control Board, Odisha, since February, 1991. He has wide experience in implementations of pollution control measures in industries and organisation of training/seminars. He has introduced Y-cable concept for real time data transmission through GPRS link from online air and water quality monitoring stations installed in industries of Odisha, India

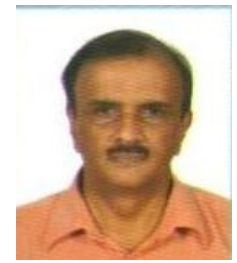

Siddhanta Das was the former member secretary, State Pollution Control Board, Odisha from March 31, 9 to March 23, 2013 and presentely as the chairman of Odisha Forest Development Corporation. He is an Indian forest service officer of 1982 batch borne in the Odisha cadre. He has done his Post-graduation in Physics from Utkal University and MBA from the Indira Gandhi National Open University. He has varied experience in areas covering Pollution Control, Disaster Management, Planning \& Policy making, Project management, Food \& Nutrition Security, Training \& Capacity Building,Strengthening of Social Capital,Integrating contribution of Civil Society Organisation in Government programmes,Forest Management with community participation, material management. With his initiative a unique pilot project for carbon sequestaration from the flue gas of Thermal Power Plant by using algae has been put in place in NALCO, Angul.

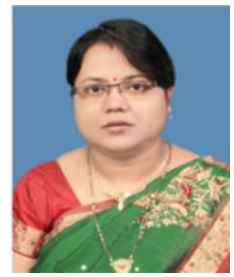

Ranjita Panda was born at Rourkela, Odisha India on May 9, 1978. She has done her B. Sc. as zoology honours from Sambalpur University, Odisha, India in 1999, M.Sc. in environmental science from Utka University, Bhubaneswar Odisha, India in 2002, M.Tech. in environmental science and engineering from Sambalpur University, Odisha, India in 2009 and Ph.D. degree from Utkal University, Bhubaneswar, Odisha, India in 2011. The major field of study include Green House Gas Monitoring and abatement Technology Industrial and Mine waste/byproductutilization, Environmental Biotechnolohy, Pollution Monitoring and EIA.

She has 10 years of work Experience in Teaching and Research in the field of Environmental Science. Previously she was working as a junior research fellow (JRF) and senior research fellow (SRF) in Institute of Minerals and Materials Technology (IMMT) CSIR, Formerly called Regional Research Laboratory (RRL) Bhubaneswar, Idisha India for six years. Now she is working as a lecturer in PG Department of Environmental Sciences Sambalpur University, Odisha, India for last four years. She has significantly contributed in the field of Environmental Sciences. Her current research was based on Bioleaching of different low grade minerals by microorganisms, Industrial waste utilization and many other aspects of Environmental Sciences 